\title{
VARIABILIDADE HORÁRIA E INTENSIDADE SAZONAL DA PRECIPITAÇÃO NO MUNICÍPIO DE HUMAITÁ-AM
}

\author{
PEDREIRA JUNIOR, Altemar Lopes - merklein3@hotmail.com \\ Universidade Federal do Amazonas / UFAM
}

QUERINO, Carlos Alexandre Santos - carlosquerino@ufam.edu.br Universidade Federal do Amazonas / UFAM

\begin{abstract}
QUERINO, Juliane Kayse Albuquerque da Silva - julianekayse@hotmail.com Universidade Federal do Amazonas / UFAM
\end{abstract}

\author{
SANTOS, Luiz Octavio Fabricio dos- luiz_octavio@outlook.com \\ Universidade Federal do Amazonas / UFAM
}

MOURA, Aryanne Resende de Melo- aryanneresende.mm@gmail.com
Universidade Federal do Amazonas / UFAM
MACHADO, Nadja Gomes- nadja.machado@blv.ifmt.edu.br Instituto Federal do Mato Grosso / IFMT

BIUDES, Marcelo Sacardi - marcelo@fisica.ufmt.br Universidade Federal do Mato Grosso / UFMT

\begin{abstract}
RESUMO: Em geral, estudos sobre precipitação focam na variabilidade mensal e anual dessa variável. Pesquisas sobre a variabilidade horária das precipitações são importantes para quantificar os efeitos da disponibilidade de água no solo, escoamento superficial e erosão. No entanto, essas informações ainda são escassas. O presente trabalho objetivou estudar a frequência relativa horária e a intensidade da precipitação no município de Humaitá-AM. Os dados de precipitação horária foram coletados na estação meteorológica do Instituto Nacional de Meteorologia INMET, localizada no Instituto Federal do Amazonas - IFAM (7.55० S e $63.070 \mathrm{~W}$ e $54 \mathrm{~m}$ ) entre julho de 2012 até julho de 2017. A frequência relativa e a variabilidade horária média sazonal foram calculadas pelo método proposto por SPIEGEL. Além disso, foi realizada análise por período seco e chuvoso e pelas estações do ano. Verificou-se que durante a estação de outono as precipitações são frequentes no período da manhã (03:00 HL e 06:00 HL), com picos secundários no início da tarde (14:00 HL) e mínimas entre 09:00 HL e 13:00 HL. No inverno, as precipitações máximas se concentram a tarde (15:00 HL e 16:00 HL) e mínimas (03:00 HL e 04:00 $\mathrm{HL}$ ). Na primavera, a precipitação é comum no decorrer da tarde e parte da noite (14:00 $\mathrm{HL}, 16: 00 \mathrm{HL}$ e 20:00 HL), e são menos constantes entre 10:00 HL e 12:00 HL. No verão, as precipitações se concentram entre 01:00 HL e 07:00 HL, com máximo as 04:00 $\mathrm{HL}$ e mínima (12:00 HL). Durante o período chuvoso (outubro a março) foram registradas máximas entre 06:00 $\mathrm{HL}$ e 07:00 HL, e mínimas entre 10:00 $\mathrm{HL}$ e 12:00 HL, enquanto que no período seco (junho a agosto) as precipitações predominam entre 06:00 HL e 08:00 HL e mínimas entre às 09:00 HL, 12:00 HL e 16:00 HL. A intensidade das precipitações foi majoritariamente classificada como fraca, seguido por eventos de categoria moderada e forte em todas as estações do ano, com exceção da primavera, quando ocorreram eventos com intensidade muito forte. Por fim, a precipitação foi mais frequente durante a manhã no outono e verão e no período da tarde na primavera e inverno.
\end{abstract}

PALAVRAS-CHAVES: Climatologia, Pluviometria, Sul do Amazonas 
HOURLY VARIABILITY AND SEASONAL INTENSITY OF THE RAINFALL IN THE MUNICIPALITY OF HUMAITÁ - AM

ABSTRACT: In general, studies about rainfall have focused on its monthly and annual variability. However, researches about the hourly variability of the rain are important to quantify the effects of water availability in the soil, surface runoff and erosions. The current paper aimed to study the relative frequency and intensity of the rainfall in the municipality of the Humaitá-AM. Hourly rainfall data were collected by the weather station belonged to the Brazilian Institute of Meteorology (INMET), installed in the Federal Institute of Amazonas - IFAM ( $7.55^{\circ} \mathrm{S}$ e $63.07^{\circ} \mathrm{W}$ e $54 \mathrm{~m}$ ) from July 2012 until July 2017. The relative frequency of the rainfall and the seasonal averaged hourly variability have been calculated by Spiegel's method. In addition also was carried out analyzes by the dry and wet period and by the seasons. In the autumn season rains are more often through the morning $(03: 00 \mathrm{HL}$ e 06:00 HL), with secondary peaks in the begin of the afternoon (14:00 HL) and minima throughout 09:00 and 13:00 HL. Rains were more often through the afternoon (15:00 and 16:00 $\mathrm{HL}$ ) and less frequent on the dawn (03:00 and 04:00 HL) during the winter. In the spring, the rainfall events are most common throughout the afternoon and part of the night (14:00 HL, 16:00 HL and 20:00 $\mathrm{HL}$ ), and are less often between 10:00 and 12:00 HL. During the summer season, the rainfall events were concentrated between 01:00 $\mathrm{HL}$ and 07:00 $\mathrm{HL}$, with maxima at 04:00HL and minima at 12:00 HL. In the wet period (October to March) were registered maxima between 06:00 $\mathrm{HL}$ and 07:00 hl, and minima between 10:00 $\mathrm{HL}$ and 12:00 $\mathrm{HL}$, in while, the dry period (June to August) the rainfall events have dominated between 06:00 $\mathrm{HL}$ and 08:00 $\mathrm{HL}$ and minima at 09:00HL, 12:00HL and 16:00 HL. Rainfalls intensity were majority classified as weak intensity event, sequenced by moderate and strong events in all the year seasons, excepted the spring when very strong events have happened. Finally, rainfall was more frequently during the morning in the autumn and summer and in the afternoon during the spring and winter.

KEYWORDS: Climatology, Rainfall, South of Amazonas.

\section{INTRODUÇÃO}

Humaitá é porta de entrada do Amazonas pelo sul do estado pelas rodovias BR-230, Transamazônica e BR 319 (PAVÃO et al., 2017). No entorno dessas rodovias concentram-se as principais atividades econômicas do município, como a pecuária, agricultura e extração de madeira (MACEDO e TEIXEIRA, 2009). Essas atividades dependem, dentre outros fatores, de informações sobre o regime de precipitação na região.

A precipitação (Ppt) é originada nas nuvens que se formam a partir da liberação do calor latente da superfície para a atmosfera (DIRMEYER e BRUBAKER, 2007). O sul da Amazônia apresenta pluviometria média anual que pode ultrapassar $1750 \mathrm{~mm}$, e em Humaitá, de acordo com a normal climatológica do INMET, esse valor é de $2193 \mathrm{~mm}$ ano-1 (INMET, 2009). Esses totais elevados são resultantes circulação atmosférica e da dinâmica dos sistemas que atuam sobre a região que, por consequência, geram as chuvas convectivas que são comuns na Amazônia (de SOUZA et al., 2005; MARENGO, 2003; MARENGO e NOBRE, 2009; ROCHA, 2010).

Os principais estudos sobre Ppt são baseados em análises da variabilidade espaço-temporal com dados mensais ou anuais (MORAES et al., 2005). Assim, há uma carência de informações sobre a distribuição horária da precipitação. Alguns estudos têm apontado que a variabilidade temporal das chuvas é de grande importância para quantificar os efeitos causados na disponibilidade de água no solo, escoamento superficial e erosão (NADARAJAH e CHOI 2007; WESTRA et al., 2013). 
A distribuição, quantidade e formas de ocorrência da Ppt podem ser variadas, e por isso, torna-se necessário determinar a intensidade para entender os tipos de precipitação e os processos (físicos e dinâmicos) que predominam na região (MARTINS e MACHADO, 2006). Entender o comportamento da sazonal e horário da precipitação serve como alternativa para solucionar diversos problemas no planejamento urbano, planejamento dos recursos hídricos e planejamento agrícola, além de prevenir catástrofes e condicionar o cotidiano da população local (SANTOS NETO et al., 2014).

Assim, o objetivo desse trabalho foi estudar a frequência relativa horária da precipitação e analisar os eventos mais intensos entre as estações do ano no município de Humaitá-AM.

\section{MATERIAL E MÉTODOS}

\section{ÁREA DE ESTUDO}

O município de Humaitá situa-se ao sul do Estado do Amazonas, à margem esquerda do Rio Madeira. Limita-se com os municípios de Tapauá e Canutama ao oeste, Manicoré ao norte, leste e oeste, e o estado de Rondônia ao sul (IBGE, 2017). Está cerca de 675 km da cidade de Manaus (AM) e 200 km da cidade de Porto Velho (RO) (Figura 1).

O município de Humaitá possui uma população estimada de 53.383 habitantes e área territorial de $33.111 \mathrm{~km}^{2}$ (IBGE, 2017). O clima da região é tropical chuvoso, com precipitação média anual de 2193,6 mm (INMET, 2009). O período chuvoso ocorre entre outubro e março e o período seco ocorre entre junho a agosto, considerando o restante dos meses o período de transição (VIDOTTO et al., 2007).

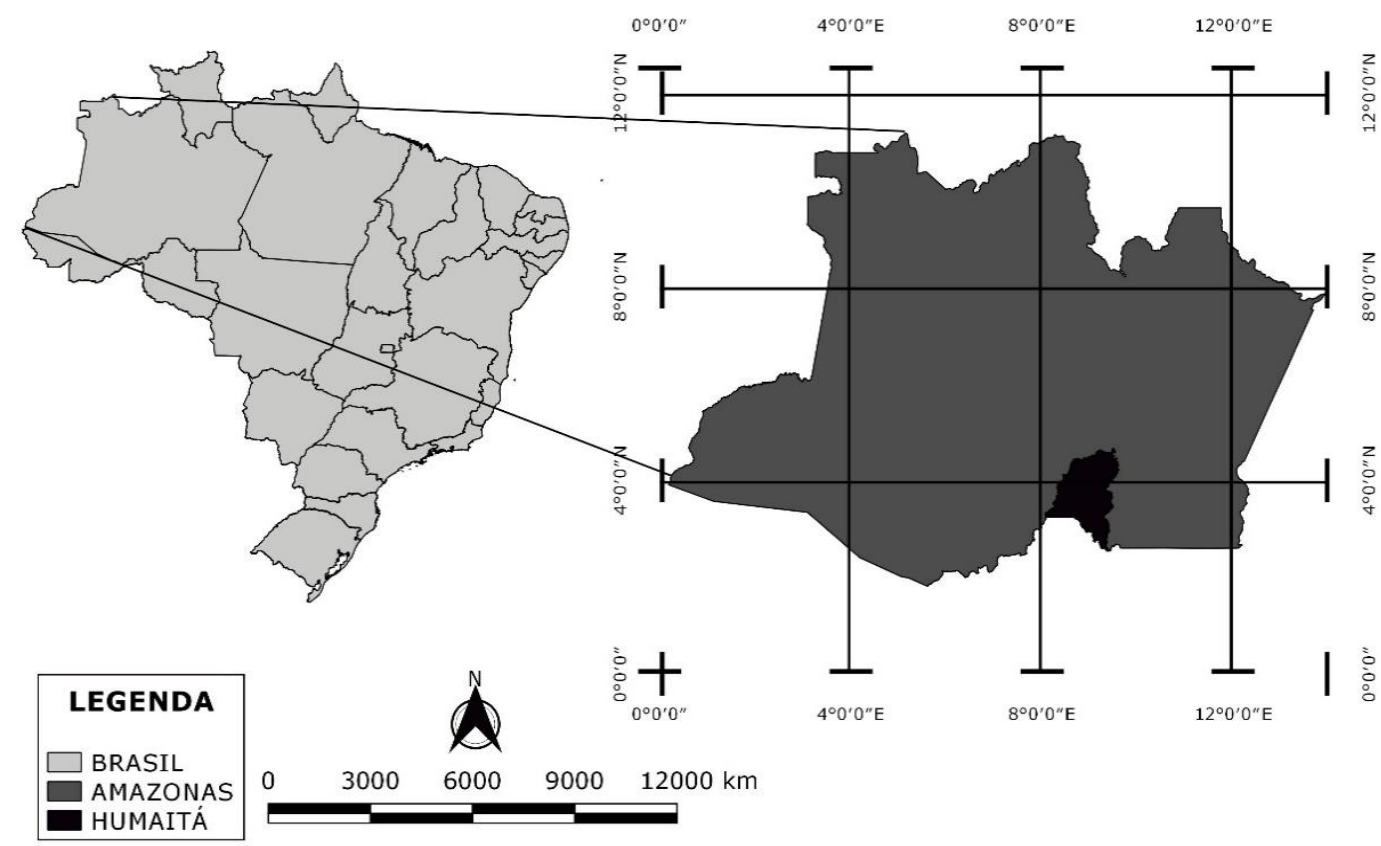

Figura 1 - Localização do município de Humaitá, no Estado do Amazonas, Brasil. 


\section{DADOS}

Os dados de Ppt foram coletados entre julho de 2012 e julho de 2015 (período com uma série de dados mais constante), por um pluviômetro (QMR102, Vaisala) na estação meteorológica automática de superfície do Instituto Nacional de Meteorologia - INMET, instalada no campus do Instituto Federal do Amazonas - IFAM (7.55 $\left.\mathrm{S}, 63.07^{\circ} \mathrm{W}, 54 \mathrm{~m}\right)$. As medidas foram realizadas a cada minuto, e integralizadas a cada hora. Todos os dados foram extraídos do Sistema de Informações Hidro - Meteorológica (SIM) do INMET (http://www.inmet.gov.br/sonabra/maps/pg_automaticas.php).

Como referência para precipitação mensal, utilizou a normal climatológica (NC) de Humaitá (INMET, 2009)

\section{PROCESSAMENTO DOS DADOS}

A frequência relativa e a variabilidade horária média sazonal foram determinadas pelo método proposto por SPIEGEL (1972) (Equação 1). Os dados de precipitação foram tabelados de acordo com o número total de eventos de precipitação registrados a cada hora pelo número total de casos em 24 horas durante o período de estudo.

$F R(\%)=\frac{F(h)}{F(24)} \times 100$

em que $F R(\%)$ é a frequência relativa da precipitação, $F(h)$ é a quantidade de eventos de precipitação que ocorreram em cada horário e $F(24)$ é o número de eventos de precipitação, acima de $0,2 \mathrm{~mm} \mathrm{~h}^{-1}$, ocorridos em um dia (24 horas).

Para melhor compreensão dos resultados, separou-se as FR da precipitação entre as estações do ano (verão, outono, inverno e primavera). Além disso, foi feita análise por período seco (junho a agosto) e chuvoso (outubro a março) (VIDOTTO et al., 2007). Classificou-se também a intensidade da chuva (IC) (Tabela 1), em chuva fraca, moderada, forte e muito forte por meio da metodologia e valores de referência adotados por Minuzzi e Sediyama (2004), o qual foi baseada na recomendação da Organização Meteorológica Mundial.

Tabela 1 - Classificação da intensidade das chuvas (IC; $\mathrm{mm} \mathrm{h}^{-1}$ ).

CATEGORIA
(IC) $\left(\mathrm{mm} \mathrm{h}^{-1}\right)$

$\begin{array}{cc}\text { Chuva Fraca } & 1,1<\text { IC } \leq 5,0 \\ \text { Chuva Moderada } & 5,1<\text { IC } \leq 25,0 \\ \text { Chuva Forte } & 25,1<\text { IC } \leq 50,0 \\ \text { Chuva Muito Forte } & \text { IC } \geq 50,1\end{array}$

FONTE: Minuzzi e Sediyama (2004). 


\section{ESTATÍSTICA DESCRITIVA DOS DADOS}

O diagnóstico estatístico descritivo foi feito pelas Médias dos Totais Mensais $\left(\bar{X}_{m}\right)$, Média dos Totais Diários $\left(\bar{X}_{D}\right)$, Erro Padrão (EP) (Equação 2), Desvio Padrão (DP) (Equação 3), Mediana (MD) (Equação 4), Variância (V) (Equação 5), Curtose (K) (Equação 6), Assimetria ( $\left.A_{s}\right)$ (Equação 7), Valores Máximos (Máx.) e Mínimos (Mín.).

$E P=\frac{D P}{\sqrt{n}}$

$\mathrm{DP}=\sqrt{\frac{\sum_{\mathrm{i}=1}^{\mathrm{n}}\left(\mathrm{X}_{\mathrm{i}}-\overline{\mathrm{X}}\right)^{2}}{\mathrm{n}-1}}$

$\mathrm{MD}=\frac{(\mathrm{n}+1)}{2}$, se $n$ for ímpar;

$\mathrm{MD}=\frac{\left(\frac{\mathrm{n}}{2}\right)+\left(\frac{\mathrm{n}}{2}+1\right)}{2}$,

$\mathrm{V}=\frac{\sum_{\mathrm{i}=1}^{\mathrm{n}}(\mathrm{Xi}-\overline{\mathrm{X}})^{2}}{\mathrm{n}-1}$

$K=\frac{1}{n} \sum\left[\frac{X i-\bar{X}}{D P}\right]^{4}-3$

se $\mathrm{n}$ for par.

$A_{s}=\frac{n}{(n-1)(n-2)} \sum\left[\frac{X_{i}-\bar{X}}{D P}\right]^{3}$

em que $n$ é o número de observações, $X_{i}$ é o valor de precipitação observado e $\bar{X}$ é a média dos valores observados de precipitação.

\section{RESULTADOS E DISCUSSÃO}

FREQUÊNCIA RELATIVA (FR) HORÁRIA E INTENSIDADE DAS CHUVAS (IC) ENTRE AS ESTAÇÕES DO ANO

Ao longo dos 5 anos, houve 2191 eventos de precipitação. Os eventos de precipitação no outono foram mais comuns no final da madrugada e início do período da manhã (Figura 2A). Os horários que ocorreram as menores FR no outono foram entre 09:00 HL e 13:00 HL, com mínimas as 12:00 HL. As chuvas com intensidade fraca foram registradas em $62 \%$ da série de dados do outono, enquanto que os eventos com categoria moderada foram $36 \%$, e de forte intensidade apenas $2 \%$ (Tabela 2 ). Contudo, não foram observadas chuvas com intensidades muito fortes. A predominância de eventos de precipitação durante a madrugada e início da manhã no outono ocorreu devido à formação de Sistemas Convectivos de Mesoescalas (SCM). Esses SCMs são oriundos da 
organização da convecção local com características cumuliformes, cobrindo ampla área espacial e apresentam chuvas moderadas (TOTA et al., 2000).

No inverno, houve predominância da precipitação no período da tarde entre 15:00 HL e 16:00 HL e ocorrências secundárias entre 07:00 HL e 08:00 HL (Figura 2B). A partir das primeiras horas da madrugada, a precipitação foi menos frequente, com pequenos registros entre as 03:00 HL e 04:00 HL (Figura 2B). Nesse período, houve predominância de eventos de fraca intensidade, seguidos por eventos de intensidade moderada e forte, e nenhum registro de chuvas muito fortes (Tabela 2).

A baixa FR e IC durante o inverno se deve pela coincidência com o período seco da região (verão Amazônico), que resulta em elevadas temperaturas, poucos eventos de precipitação e, consequentemente, baixos níveis de umidade. O período seco na região ocorre porque a circulação da baixa troposfera desloca-se mais ao norte do cavado equatorial o que induz o deslocamento do ramo descendente da Célula de Hadley sobre a Amazônia devido à máxima convecção tropical deslocar-se para a América Central neste período (FISCH, 1995; de SOUZA e AMBRIZZI, 2004). Contudo, o escoamento em baixos níveis da atmosfera (entre 925 e $700 \mathrm{hPa}$ ), oriundo do Oceano Atlântico, favorece o transporte de umidade na região central e sul da Amazônia durante este período o que garante condições necessárias para convecção local durante a tarde e explica a disparidade em relação aos demais períodos do dia (VITORINO et al., 2006; MARENGO e NOBRE, 2009; REBOITA et al., 2010; SANTOS NETO et al., 2014). A maior porcentagem de eventos com intensidade forte no inverno é resultado da penetração de frentes de massas de ar fria na região, localmente conhecidas como friagens (FISCH, 1995; MARENGO et al., 1997; BONATTI, 2006; RIBEIRO, 2012). No inverno austral, é comum as frentes frias penetrarem ao sul do Amazonas e atingirem baixas latitudes, provocando precipitação e queda de temperatura (SANTOS NETO, 2014). Ainda segundo o autor, a chegada de uma frente fria em julho de 2013, foi responsável pela precipitação de $24,2 \mathrm{~mm}$ em uma hora em Porto Velho, RO, distante $200 \mathrm{~km}$ do local de estudo.

Na primavera, a precipitação se concentrou ao longo de todo o período da tarde e início da noite (Figura 2C). A menor FR na primavera ocorreu entre 10:00 HL e 12:00 HL. A Intensidade das Chuvas (IC) na primavera apresenta predominância de chuvas fracas, seguidas de moderadas, fortes e apenas $0,3 \%$ de ocorrência de chuvas muito fortes (Tabela 2). A primavera é caracterizada pelo início da atividade convectiva devido às altas temperaturas e ao forte aquecimento superficial que pode acarretar na ocorrência de precipitação acima de $50 \mathrm{~mm} \mathrm{~h}^{-1}$ durante a tarde. Nessa estação observa-se também incursões de sistemas frontais que chegam até a região sul do Amazonas, início dos episódios de ZCAS e o início dos Sistemas de Monção da América do Sul (SMAS) que gera profunda convecção e, deste modo, inicia o período de chuvas na região sul da Amazônia (GAN et al., 2004; ROLIM et al., 2006).

No verão austral, a precipitação se distribuiu de forma mais uniforme ao longo do dia (Figura 2D). As maiores FR foram observadas a 01:00h e 07:00 HL com máxima frequência às 04:00 HL da manhã. As FR secundárias ocorreram a tarde entre 13:00 HL e 15:00 HL e as menores FR ocorreram às 12:00 HL. A intensidade de chuvas no verão foi fraca, seguidas por chuvas de intensidade 
moderada e forte, sem registro de eventos de intensidade muito forte (Tabela 2).

A distribuição de precipitação ao longo do dia no verão se deve à coincidência com período de chuvas da região, o qual é governado pela atuação do Sistema de Monção da América do Sul (GAN et al., 2004), pela ZCAS e pela Alta da Bolívia (AB). A ZCAS se estende da Amazônia até o sudeste do país, e provoca altos volumes de precipitação devido a intensa atividade convectiva sobre a região (SEIXAS, 2011).

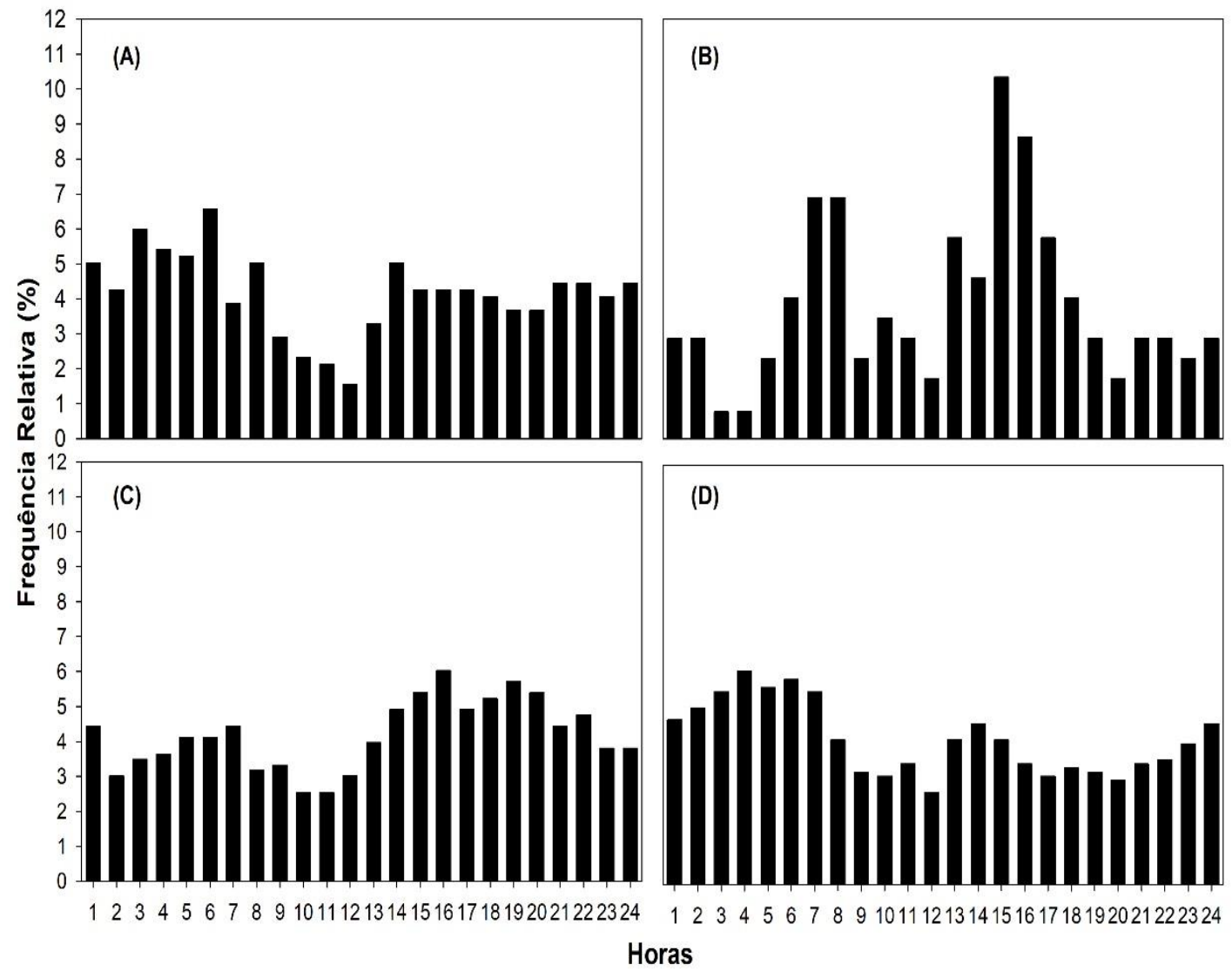

Figura 2 - Frequência Relativa (FR) Horária da precipitação entre as estações de outono $(A)$, inverno (B), primavera (C) e verão (D), no município de Humaitá - AM, durante o período de julho de 2012 a julho 2017.

Tabela 2 - Intensidade das chuvas (IC; \%) durante as estações do ano entre o período de julho de 2012 a julho de 2017 no município de Humaitá-AM.

\begin{tabular}{ccccc}
\hline $\begin{array}{c}\text { Estações } \\
\text { do Ano }\end{array}$ & $\begin{array}{c}\text { (IC) Fraca } \\
(\%)\end{array}$ & $\begin{array}{c}\text { (IC) Moderada } \\
(\%)\end{array}$ & $\begin{array}{c}\text { (IC) Forte } \\
(\%)\end{array}$ & $\begin{array}{c}\text { (IC) Muito } \\
\text { Forte (\%) }\end{array}$ \\
\hline Outono & 62 & 36 & 2 & 0 \\
Inverno & 63 & 30 & 7 & 0 \\
Primavera & 61,5 & 34,6 & 3,5 & 0,3 \\
Verão & 67 & 29 & 4 & 0 \\
\hline
\end{tabular}




\section{FREQUÊNCIA RELATIVA (FR) HORÁRIA DURANTE OS PERÍODOS CHUVOSO E SECO}

Das 14808 horas analisadas durante o período chuvoso (outubro a março), ocorreram 1913 eventos de precipitação acima de 0,2 $\mathrm{mm} \mathrm{h}^{-1}$. Entretanto, no período seco (junho, julho e agosto) em um total de 5580 horas, foram registradas apenas 130 ocorrências. No período chuvoso a precipitação se distribuiu ao longo de todo o dia. A FR horária máxima ocorreu entre 06:00 e 07:00 HL e ocorreu um segundo pico pelo período da tarde às 14:00 HL. Os horários de menores FR foram entre 10:00 HL e 12:00 HL (Figura 3A). No período seco, a maior FR ocorreu no período da tarde, com pico secundário no início da manhã (Figura 3B). Em ambos os caso, da estação seca, a Ppt está associada à entrada de frentes frias que chegam ao sul da Amazônia, o que contribui para modificar a dinâmica da atmosfera, pela alteração da condição de estabilidade para situação de instabilidade nesse período (FISCH, 1995).

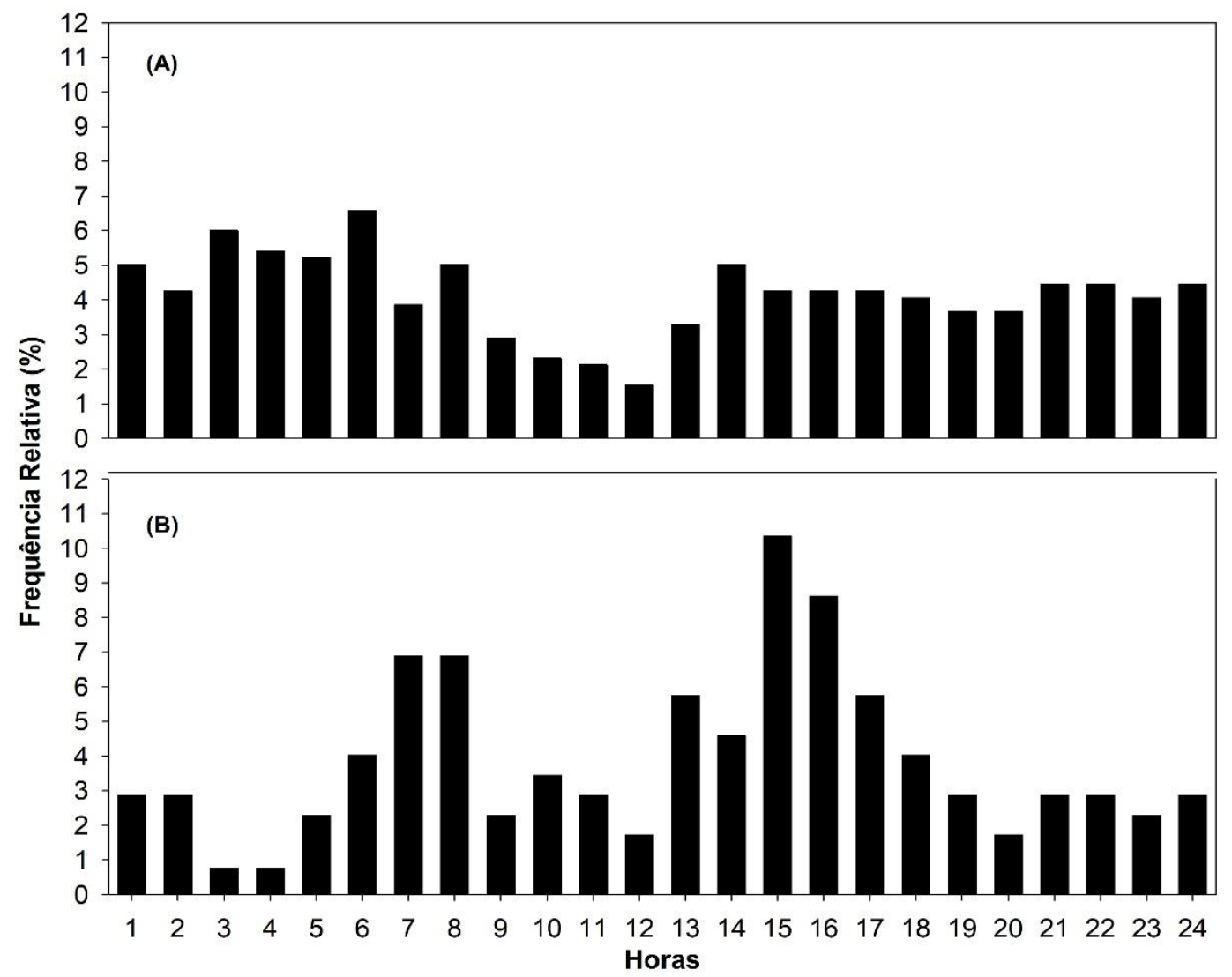

Figura 3 - Frequência relativa (FR) horária da precipitação no decorrer do peíodo chuvoso (A) e período seco (B), no município de Humaitá - AM, entre os anos de 2012 e 2017.

Os resultados desse estudo corroboram com os de Santos Neto et al. (2014) em Porto Velho - RO, distante $200 \mathrm{~km}$ de Humaitá-AM. Os autores observaram que como o período chuvoso coincide com a estação de verão e parte da primavera, ocorre altos índices de precipitação, com maior FR durante a manhã e pico secundário a tarde por influência da ZCAS e do SMAS (CARVALHO et al., 2010; GAN et al., 2004). 
As FRs acumuladas em cada período do dia, tanto ao longo da estação chuvosa como da seca, foram maiores durante a madrugada (Tabela 3). Entretanto, as menores FR no período chuvoso ocorreram de manhã. Embora, para o período seco se concentraram a noite.

Tabela 3 - Frequência relativa (FR; \%) da precipitação por períodos da madrugada (01:00h - 06:00h), manhã (07:00h - 12:00h), tarde (13:00h - 18h:00) e noite (19:00h 24:00h) nos períodos chuvoso e seco.

\begin{tabular}{cccc}
\hline \multicolumn{2}{c}{ Período Chuvoso } & \multicolumn{2}{c}{ Período seco } \\
\hline Período do Dia & FR (\%) & Período do Dia & FR (\%) \\
\hline Madrugada & 28,7 & Madrugada & 30,9 \\
Manhã & 21,1 & Manhã & 22,4 \\
Tarde & 25,6 & Tarde & 27,9 \\
Noite & 24,5 & Noite & 18,8 \\
\hline
\end{tabular}

A maior quantidade de Ppt na madrugada e a tarde é resultado de Sistemas Convectivos de Mesoescala (SCM), oriundos da organização convectiva local, devido ao aquecimento da superfície ao longo do dia que favorece o transporte de calor latente e umidade (TOTA et al., 2000; ADAMS et al., 2009). Esses SCM abrangem uma grande área espacial com chuvas de intensidade fraca e moderada.

Outros sistemas meteorológicos que também contribuem para ocorrência de precipitação durante esses períodos do dia são: as Linhas de Instabilidade (LI), que se desenvolvem no litoral e deslocam-se no sentido oeste, causando precipitação no período da tarde e noite (RICKENBACH, 2004), a ZCAS resultante da interação entre diversos sistemas meteorológicos e ocasiona um período prolongado de chuva frequente e volumosa, e os sistemas de meso e larga escala que estão relacionados com a atuação de sistemas frontais que chegam até a região Amazônica (GAN et al., 2004; DE SOUZA et al., 2016).

\section{ANÁLISES ESTATÍSTICAS}

A Ppt foi $24,4 \%$ superior a NC no mês de março (Tabela 4 ). O mês de janeiro foi o que registrou o volume máximo de Ppt. As menores médias mensais foram observadas no mês de julho e agosto. O Desvio Padrão (DP), Variância (V) e a Mediana (MD), foram elevadas para os meses com maiores volumes de precipitação. O DP e a $\mathrm{V}$ indicaram que os dados estão dispersos da média mensal, no qual o mês de fevereiro apresenta uma maior variação e junho a menor. A MD demonstrou que pelo menos metade das observações para cada mês são inferiores ou iguais, ou superior ou iguais. No entanto, a série de dados apresentou uma distribuição positiva, pois todos os valores da mediana foram inferiores à média diária (Tabela 4).

Todos os meses apresentam curtose com uma distribuição positiva (curva leptocúrtica), ou seja, verifica-se que há uma relativa regularidade dos dados de precipitação ao longo dos meses. O período chuvoso da região compreendeu entre outubro e março $(O, N, D, J, F$ e $M)$, e o período de estiagem compreendeu entre junho e agosto, sendo julho o mês com menor índice pluviométrico, 9,54\% superior a NC publicada pelo Instituto Nacional de Meteorologia (INMET, 2009). 
Tabela 4 - Estatística descritiva dos dados de precipitação médios diários a partir de julho de 2012 a julho de 2017. $\bar{X}_{m}$ : Média Mensal; $\bar{X}_{D}$ : Média Diária; $\Sigma$ : Soma; EP: Erro Padrão; MD: Mediana; DP: Desvio Padrão; V: Variância; K: Curtose; As: Assimetria; Mín: Mínimo; Máx: Máximo.

\begin{tabular}{ccccccccccc}
\hline Meses & $\overline{\mathbf{X}} \mathbf{m}$ & $\overline{\mathbf{X}}_{\mathbf{D}}$ & $\mathbf{E P}$ & $\mathbf{M D}$ & $\mathbf{D P}$ & $\mathbf{V}$ & $\mathbf{K}$ & As & Mín. & Máx. \\
\hline Jan & 391,3 & 7,2 & 2,2 & 6,8 & 19,6 & 382,6 & 3,3 & 1,9 & 0,2 & 86,8 \\
Fev & 354,3 & 7,0 & 2,8 & 5,0 & 23,2 & 539,2 & 8,4 & 2,7 & 0,2 & 123,4 \\
Mar & 404,7 & 7,4 & 2,5 & 4,5 & 21,9 & 479,5 & 6,4 & 2,4 & 0,2 & 117,0 \\
Abr & 230,2 & 7,3 & 1,7 & 3,4 & 14,0 & 196,7 & 8,6 & 2,9 & 0,2 & 73,6 \\
Mai & 128,4 & 7,1 & 1,1 & 0,7 & 7,9 & 61,9 & 1,4 & 1,6 & 0,2 & 29,0 \\
Jun & 32,7 & 5,8 & 0,7 & 0,2 & 3,6 & 13,1 & 5,3 & 2,3 & 0,2 & 14,6 \\
Jul & 21,8 & 6,5 & 0,9 & 0,2 & 3,8 & 14,3 & 13,8 & 3,6 & 0,2 & 15,4 \\
Ago & 26,4 & 6,9 & 3,0 & 2,0 & 6,7 & 45,4 & 3,3 & 1,8 & 0,2 & 16,8 \\
Set & 83,0 & 6,7 & 3,3 & 2,7 & 14,8 & 219,9 & 3,9 & 2,2 & 0,2 & 49,4 \\
Out & 166,9 & 6,7 & 2,1 & 3,4 & 14,5 & 209,8 & 3,8 & 2,1 & 0,2 & 62,8 \\
Nov & 187,7 & 6,7 & 1,5 & 2,9 & 13,3 & 177,1 & 7,9 & 2,8 & 0,2 & 63,6 \\
Dez & 302,7 & 6,8 & 1,5 & 4,2 & 13,6 & 185,3 & 9,5 & 2,6 & 0,2 & 81,0 \\
\hline
\end{tabular}

\section{CONCLUSÕES}

A precipitação horária no município de Humaitá durante o período analisado (2012 a 2017) foi mais frequente durante a manhã nas estações de outono e verão, e mais frequentes no período da tarde nas estações da primavera e inverno.

A intensidade das chuvas que teve maior predominância ao longo de todo o ano foi fraca, seguido de moderada e forte. Ao longo de todo período analisado houve apenas um evento com intensidade muito forte, que ocorreu na estação da primavera.

Este estudo permitiu demonstrar os horários de predominância dos eventos de precipitação e suas intensidades no município de Humaitá-AM. Espera-se que os resultados desse estudo possam ser úteis para o desenvolvimento do município, ou ainda, em pesquisas que demandem este tipo de informação.

Por fim, ressalta-se que estudos futuros são importantes por um período maior e continuo de dados de precipitação, bem como, abranger mais municípios do sul do Amazonas e, assim, propiciar um melhor conhecimento da interação da precipitação com outras variáveis meteorológicas. 


\section{AGRADECIMENTOS}

A Universidade Federal do Amazonas (UFAM), ao Programa de Apoio à Iniciação Cientifica - PAIC UFAM 2017/2018 pela concessão da bolsa de iniciação científica PIBIC, projeto número PIB-E/0173/2017, e ao Conselho Nacional de Desenvolvimento Científico e Tecnológico (CNPq) pelas bolsas 303625/2015-5; 310879/2017-5. Os autores também agradecem ao Instituto Nacional de Meteorologia - INMET, pela disponibilidade dos dados.

\section{REFÊRÊNCIAS BIBLIOGRÁFICAS}

ADAMS, D. K.; SOUZA, E. P.; COSTA, A. A. Convecção úmida na Amazônia: implicações para modelagem numérica. Revista Brasileira de Meteorologia, v. 24, p. 168-178, 2009.

BONATTI, J. P. Estudo observacional de caso da energética modal e de modelagem do fenômeno friagem na Amazônia. Anais do XIV Congresso Brasileiro de Meteorologia, 2006.

CARVALHO, L. M. V.; SILVA, A. E.; JONES, C.; LIEBMANN, B.; DIAS, P. L. S.; ROCHA, H. R. Moisture transport and intraseasonal variability in the South America monsoon system. Climate Dynamics, v. 34, p. 1-20, 2010.

DE SOUZA, E. B.; AMBRIZZI, T. Pentad precipitation climatology over Brazil and the associated atmospheric mechanisms. Climanálise, v. 2, n. 1, p. 1-20, 2004.

DE SOUZA, E. B.; CARMO, A. M. C.; MORAES, B. C.; NACIF, A.; FERREIRA, D. B. S.; ROCHA, E. J. P.; SOUZA, P. J. O. P. Sazonalidade da Precipitação Sobre a Amazônia Legal Brasileira: Clima Atual e Projeções Futuras Usando o Modelo REGCM4. Revista Brasileira de Climatologia, V. 18, 2016.

DE SOUZA, E. B.; KAYANO, M. T.; AMBRIZZI, T. Intraseasonal and submonthly variability over the eastern Amazon and northeast Brazil during the autumn rainy season. Theoretical and Applied Climatology, v. 81, p. 177-191, 2005.

DIRMEYER, P. A.; BRUBAKER, K. L. Characterization of the Global Hydrologic Cycle from a Back-Trajectory Analysis of Atmospheric Water Vapor. J. Hydrometeorology, v. 8, p. 20-37, 2007.

FISCH, G. Camada Limite Amazônica: aspectos observacionais e de modelagem. Tese (Doutorado), Instituto Nacional de Pesquisas Espaciais - INPE, São José dos Campos, São Paulo. 125p. 1995.

GAN, M. A.; KOUSKY, V. E.; ROPELEWSKI, C. F. The South America monsoon circulation and its relashionship to rainfall over West-Central Brazil. Journal of Climate, v. 17, p. 47-66, 2004.

IBGE - Instituto Brasileiro de Geografia e Estatística. Amazonas. 2017.

INMET - Instituto Nacional de Meteorologia. Normais Climatológicas do Brasil 1961-1990. Brasília, DF. 465p. 2009.

MACEDO, M. A.; TEIXEIRA, W. Sul do Amazonas, nova fronteira agropecuária? O caso do município de Humaitá. XIV Simpósio Brasileiro de Sensoriamento Remoto. Natal, p. 5933-5940, 2009. 
MARENGO, J. A. Condições climáticas e os recursos hídricos no norte brasileiro. In: TUCCI, C. E. M.; BRAGA, B. (Org.). Clima e Recursos Hídricos no Brasil. Coleção ABRH, 2003.

MARENGO, J. A.; NOBRE, C. Clima da região Amazônica. In: CAVALCANTI, I. F. A. et al. Tempo e clima no Brasil. São Paulo: Oficina de Textos, p. 197-212, 2009.

MARENGO, J. A.; NOBRE, C. A.; CULF, A. D. 1997. Climatic Impacts of "Friagens" in forested and deforested areas of the Amazon Basin. Journal of Applied Meteorology, 36: 1553-1566.

MARTINS, R. C. G.; MACHADO, L. A. T. Análise das Características Microfísicas da Precipitação na Estação Chuvosa da Região Amazônica Durante o Experimento WET-AMC. Anais do XIV Congresso Brasileiro de Meteorologia. Florianópolis-SC: SBMET, 2006.

MINUZZI, R. B.; SEDIYAMA, G. C. Influência da topografia na precipitação: uma análise estatística e via imagens de satélite. Congresso Brasileiro de Meteorologia, Fortaleza, 2004.

MORAES, B. C.; COSTA, J. M.; COSTA, A. C. L.; COSTA, M. H. Variação espacial e temporal da precipitação no estado do Pará. ACTA Amazonica, v. 35, p.207214, 2005.

NADARAJAH, S.; CHOI, D. Maximum daily rainfall in South Korea. Journal of Earth System Science, v. 116, p. 311-320, 2007.

PAVÃO, L. L.; QUERINO, C. A. S.; BIUDES, M. S.; PAVÃO, V. M.; QUERINO, J. K. A. S.; MACHADO, N. G.; BENEDITTI, C. A.; PEIXOTO, K. L. G. Distribuição espaço-temporal da temperatura da superfície urbana do município de Humaitá - sul do Amazonas. RA'E GA (UFPR), v. 42, p. 210-224, 2017.

REBOITA, M. S.; GAN, M. A.; DA ROCHA, R. P.; AMBRIZZI, T. Regimes de Precipitação na América do Sul: Uma Revisão Bibliográfica. Revista Brasileira de Meteorologia, v.25, p. 185-204, 2010.

RICKENBACH, T. M. Nocturnal cloud systems and the diurnal cariation of clouds and rainfall in Southwestern Amazonia. Monthly Weather Review, v. 132, p. 1201-1219, 2004.

ROCHA, A. A. A. A relação do perfil vertical do vapor d água com a convecção profunda na Amazônia. Dissertação (Mestrado), Instituto Nacional de Pesquisas da Amazônia - INPA, Manaus, 2010.

ROLIM, P. A. M.; SANTOS, D. M.; ROCHA, E. J. P.. Variabilidade da Precipitação na Amazônia: Implicações Socioeconômicas. In: XIV Congresso Brasileiro de Meteorologia, Florianópolis - SC. v. 1, 2006.

RIBEIRO, I. L. As incursões de ar frio no estado do Amazonas. Dissertação (Mestrado) - Programa de Pós-graduação em Geografia, Universidade Federal do Amazonas, Manaus, Amazonas. 88f. 2012.

SANTOS NETO, L. A. Variabilidade da precipitação horária em Porto Velho - RO e suas tendências anuais e sazonais. Dissertação (Mestrado) - Programa de Pós-graduação em Desenvolvimento Regional e Meio Ambiente, UNIR, Porto Velho, Rondônia. 71f. 2014. 
SANTOS NETO, L. A.; MANIESI, V.; SILVA, M. J. G.; QUERINO, C. A. S.; LUCAS, E. W. M.; BRAGA, A. P.; ATAÍDE, K. R. P. Distribuição horária da precipitação em Porto Velho-RO no período de 1998 - 2013. Revista Brasileira de Climatologia, v.14, 2014.

SEIXAS, I. M. Geotecnologias aplicadas ao estudo de precipitação e desmatamento na porção sul da Amazônia. Dissertação (Mestrado) - Programa de Pós-Graduação em Geografia, 2011.

SPIEGEL, M.R. Estatística. São Paulo: McGraw-Hill do Brasil, 1972.

TOTA, J.; FISCH, G.; FUENTES, J.; OLIVEIRA, P. J.; GARSTANG, M.; HEITZ, R.; SIGLER, J. Análise da variabilidade diária da precipitação em área de pastagem para a época chuvosa de 1999 - projeto TRMM/LBA. ACTA Amazônica, v. 30, n.4, p. 629-639, 2000.

VIDOTTO, E.; PESSENDA, L. C. R.; RIBEIRO, A.S.; FREITAS, H. A.; BENDASSOLLI, J. A. Dinâmica do ecótono floresta-campo no sul do estado do Amazonas no Holoceno, através de estudos isotópicos e fitossociológicos. ACTA Amazônica, v. 37, p. 385-400, 2007.

VITORINO, M.I.; DA SILVA, M.M.; DA SILVA, R.P. Eventos de Precipitação Durante a Estação Seca no Estado do Amazonas. In: Congresso Brasileiro de Meteorologia, 14, Florianópolis-SC. Anais... Florianópolis: SBMET, CD-ROM, 2006.

WESTRA, S.; ALEXAQNDER, L. V.; ZWIERS, F. W. Global in creas ingtrends in annual maximum daily precipitation. Journal of Climate, v.26, n.11, p. 39033918, 2013. 